\title{
Counterpropagating Radiative Shock Experiments on the Orion Laser
}

\author{
F. Suzuki-Vidal, ${ }^{1, *}$ T. Clayson, ${ }^{1}$ C. Stehlé, ${ }^{2}$ G. F. Swadling, ${ }^{1, \dagger}$ J. M. Foster, ${ }^{3}$ J. Skidmore, ${ }^{3, \S}$ P. Graham, ${ }^{3}$ G. C. Burdiak, ${ }^{1}$ \\ S. V. Lebedev, ${ }^{1}$ U. Chaulagain, ${ }^{2,+}$ R. L. Singh, ${ }^{2}$ E. T. Gumbrell,,${ }^{3, \|}$ S. Patankar, ${ }^{3, \dagger}$ C. Spindloe, ${ }^{4}$ J. Larour, ${ }^{5}$ M. Kozlova, ${ }^{6}$ \\ R. Rodriguez, ${ }^{7}$ J. M. Gil, ${ }^{7}$ G. Espinosa, ${ }^{7}$ P. Velarde, ${ }^{8}$ and C. Danson ${ }^{3}$ \\ ${ }^{1}$ Blackett Laboratory, Imperial College London, London SW7 2BW, United Kingdom \\ ${ }^{2}$ Laboratoire d'Etude du Rayonnement et de la Matière en Astrophysique et Atmosphères (LERMA), \\ Centre National de la Recherche Scientifique (CNRS), Observatoire de Paris, PSL Research University, \\ Université Pierre et Marie Curie (UPMC), Sorbonne University, 4 Place Jussieu, 75252 Paris, France \\ ${ }^{3}$ Atomic Weapons Establishment (AWE), Aldermaston, Reading, West Berkshire RG7 4PR, United Kingdom \\ ${ }^{4}$ Science and Technology Facilities Council, Rutherford Appleton Laboratory, \\ Harwell Campus, Chilton, Didcot, Oxon OX11 OQX, United Kingdom \\ ${ }^{5}$ Laboratoire de Physique des Plasmas (LPP), Centre National de la Recherche Scientifique (CNRS), Ecole Polytechnique, \\ Université Pierre et Marie Curie, Université Paris-Saclay, Sorbonne University, \\ PSL Research University, 4 Place Jussieu, 75252 Paris, France \\ ${ }^{6}$ Extreme Light Infrastructure (ELI), Institute of Plasma Physics, Czech Academy of Sciences, 18200 Prague 8, Czech Republic \\ ${ }^{7}$ Departamento de Fisica, Universidad de Las Palmas de Gran Canaria, 35003 Las Palmas de Gran Canaria, Las Palmas, Spain \\ ${ }^{8}$ Instituto de Fusion Nuclear, Universidad Politecnica de Madrid, 28040 Madrid, Spain
}

(Received 11 November 2016; published 2 August 2017)

\begin{abstract}
We present new experiments to study the formation of radiative shocks and the interaction between two counterpropagating radiative shocks. The experiments are performed at the Orion laser facility, which is used to drive shocks in xenon inside large aspect ratio gas cells. The collision between the two shocks and their respective radiative precursors, combined with the formation of inherently three-dimensional shocks, provides a novel platform particularly suited for the benchmarking of numerical codes. The dynamics of the shocks before and after the collision are investigated using point-projection x-ray backlighting while, simultaneously, the electron density in the radiative precursor was measured via optical laser interferometry. Modeling of the experiments using the 2D radiation hydrodynamic codes NYM and PETRA shows very good agreement with the experimental results.
\end{abstract}

DOI: 10.1103/PhysRevLett.119.055001

Radiative shocks are formed when shocked matter becomes hot enough that radiative energy transfer changes the shock structure. Radiative shocks are ubiquitous in astrophysical phenomena including supernovae [1] and protostellar jets [2]. Photons escaping from the shock can heat and ionize the unshocked medium ahead of it, leading to the formation of a radiative precursor $[3,4]$. The traditional study of radiative shocks has relied on theory [5] and numerical simulations for the interpretation of astrophysical phenomena (see, e.g., Ref. [6]) and experimental data [7-10], which requires the addition of nonlocal radiative transport to multidimensional hydrodynamics. The growth of instabilities and other nonideal effects can further modify the physics; thus, experimental data are essential in order to test these models and improve our understanding of the physics of radiative shocks.

Published by the American Physical Society under the terms of the Creative Commons Attribution 4.0 International license. Further distribution of this work must maintain attribution to the author(s) and the published article's title, journal citation, and DOI.
Experiments to produce radiative shocks are typically performed with high-power lasers, which can produce and accelerate plasma flows to velocities of $\sim 10-100 \mathrm{~km} / \mathrm{s}$ (see the references in Refs. [11,12]). One experimental approach to studying such radiative effects consists of producing radiative blast waves by focusing lasers onto a gas-embedded pin $[13,14]$ or into a puffed cluster gas [15-17]. In these cases, the shocks decelerate following a Sedov-Taylor trajectory. An alternative experimental approach consists of focusing lasers onto a foil which, due to laser ablation pressure, acts as a piston that continuously pushes and compresses a static gas inside a tube or gas cell. In order to maximize the radiative effects, the experiments are typically performed in high atomic-number gases such as xenon, at pressures $\lesssim 1$ bar [18-23]. Results from these piston-driven experiments show the formation of quasiplanar radiative shocks, albeit perturbed by the interaction of the shock with the walls of the tube at velocities $\gtrsim 100 \mathrm{~km} / \mathrm{s}$ [24]. These experiments have led to novel applications, particularly in the area of laboratory astrophysics, e.g., reverse-radiative shocks for studies of accretion in cataclysmic variables [25].

In this Letter we report on new experiments designed to investigate the formation of piston-driven radiative shocks. 
The experiments were designed so that the shocks are able to propagate both axially and radially, preventing in this way any interaction of the shocks with the internal walls of the cells and thus leading to a quasispherical shock geometry [26]. In addition, the collision and interaction between two counterpropagating radiative shocks and their respective radiative precursors is introduced as a radiationhydrodynamics platform particularly suited for laboratoryastrophysics studies (e.g., colliding supernova remnants $[27,28])$ and numerical benchmarking.

The experimental setup (Fig. 1) consisted of octogonal gas cells with plastic disks attached to opposite ends acting as pistons. The pistons were made up of $25 \mu \mathrm{m}$ thick $\mathrm{CH}$ $\left(\rho_{\mathrm{CH}}=0.9 \mathrm{~g} / \mathrm{cm}^{3}\right)$ with a $50 \mu \mathrm{m}$ thick $\mathrm{CH}-\mathrm{Br}\left(\rho_{\mathrm{CH}-\mathrm{Br}}=\right.$ $1.53 \mathrm{~g} / \mathrm{cm}^{3}$ ) attached to the inside surface to prevent earlytime preheating of the gas from $\mathrm{x}$ rays from the interaction of the drive lasers with the $\mathrm{CH}$. This ensured that the formation of a radiative precursor comes predominantly from the heating of the compressed gas in the shock.

The experiments were conducted on the Orion laser [29]. The shocks were driven using four laser beams that were focused onto each piston simultaneously. Each beam $(\sim 400 \mathrm{~J}, \lambda=351 \mathrm{~nm}, 1 \mathrm{~ns}$ pulse duration) had a flattopped spatial profile with a $\sim 600 \mu \mathrm{m}$ spot diameter and thus a laser intensity of $\sim 5 \times 10^{14} \mathrm{~W} / \mathrm{cm}^{2}$. Side-on diagnostic access was achieved through two pairs of opposite windows sealed with gas-tight filters suitable for optical and $\mathrm{x}$-ray diagnostics. The gas cells were filled with xenon to a pressure of $P_{0} \sim 0.3$ bar $\left(\rho_{0} \sim 1.6 \mathrm{mg} / \mathrm{cm}^{3}\right)$.

The diagnostic setup is presented in Fig. 1(c). One pair of windows was used for point-projection $\mathrm{x}$-ray backlighting (XRBL) imaging of the shocks driven by additional lasers ( $\sim 50 \mathrm{~J}, 500 \mathrm{ps}$ pulse duration) focused onto a $5 \mu \mathrm{m}$ thickness iron foil supported on a $20 \mu \mathrm{m}$ diameter pinhole that provided spatial resolution [30]. This is comparable to the resolution due to motion blurring $(\sim 40 \mu \mathrm{m})$ for the XRBL laser pulse duration and a typical shock velocity of
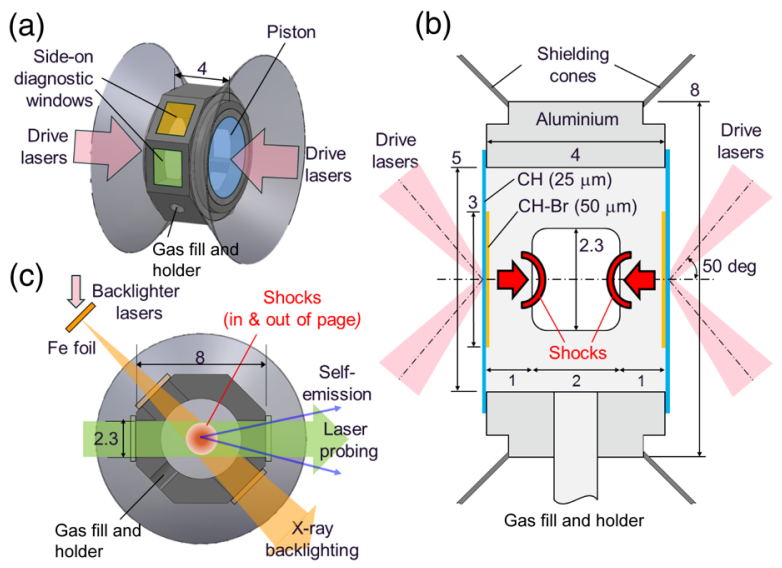

FIG. 1. Octogonal gas cells (nominal dimensions in $\mathrm{mm}$ ). (a) 3D rendering. (b) Side-on, cut view. (c) Face-on view and diagnostics. $v_{s} \sim 80 \mathrm{~km} / \mathrm{s}$. The resulting emission is dominated by iron He- $\alpha$ transitions (6.7 keV photons) [31] and was recorded onto image plates with a magnification of $\sim 11 \times$.

The second pair of windows were used to perform optical laser interferometry in a Mach-Zehnder configuration with a $\sim 300 \mathrm{~mJ}, \lambda=532 \mathrm{~nm}, 50 \mathrm{~ns}$ pulse duration, $\sim 35 \mathrm{~mm}$ beam diameter laser. Two optical streak cameras (100 ns sweep time) recorded interferometry and optical self-emission along the axis of propagation of the shocks. In addition, four gated optical intensifiers (GOIs) recorded time-resolved, 2D interferometry images of the shocks at four different times per experiment.

Figures 2(a)-2(c) show results from XRBL at 25, 30, and $35 \mathrm{~ns}$. The shocks are seen as round-shaped features coming into the field of view of the windows from each side, with darker tones representing stronger x-ray absorption (i.e., higher mass density). Their head-on collision is seen at $30 \mathrm{~ns}$ followed by the formation of reverse shocks as dense structures at the center of the window at $35 \mathrm{~ns}$. These results indicate a shock velocity of $v_{\mathrm{s}} \sim 75 \pm 25 \mathrm{~km} / \mathrm{s}$ (i.e., a shock displacement of $\sim 0.25-0.5 \mathrm{~mm}$ in $5 \mathrm{~ns}$ ) and a reverse shock velocity on the order of $v_{\mathrm{rs}} \sim 30 \mathrm{~km} / \mathrm{s}$.

Figures 2(d)-2(f) show 2D axisymmetric simulations with the radiation-hydrodynamics codes NYM and PETRA using the same initial experimental conditions as the experiments ( $2 \mu \mathrm{m}$ resolution). NYM [32] is a Lagrangian code with multigroup implicit Monte Carlo x-ray transport and full laser-interaction physics used to model the laserpiston interaction. These simulations were linked and mapped to the Eulerian code PETRA (typically after $5 \mathrm{~ns}$ ) [33], using multigroup x-ray diffusion to study the late-time
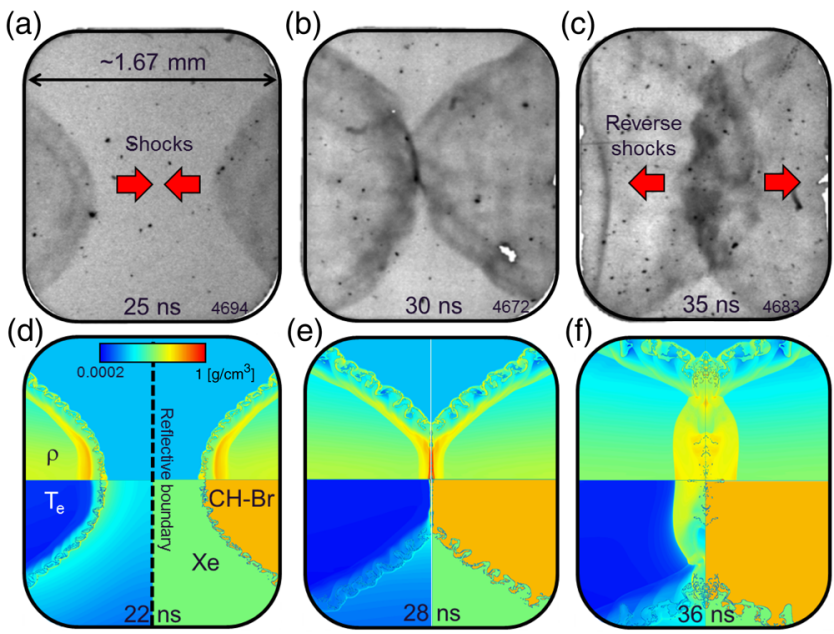

FIG. 2. Counterpropagating shock dynamics at different times from (a)-(c) experimental x-ray backlighting and (d)-(f) 2D numerical simulations. Each simulation image shows mass density (top half, log scale), electron temperature (bottom-left quadrant, linear scale) and materials (bottom-right quadrant). The color bar used to represent mass density in (d) also displays linear values of electron temperature in the ranges (d) $0-35 \mathrm{eV}$, (e) $0-60 \mathrm{eV}$, and (f) $0-40 \mathrm{eV}$. 
plasma behavior. The opacities and equations of state for the multimaterial piston and xenon were taken from SESAME tables. The counterpropagating shock collision was simulated using a fully reflective boundary at the center of the diagnostic window [shown schematically in Fig. 2(d)] for the plasma flow and radiation.

The simulations accurately reproduce the overall shock dynamics with an uncertainty of up to $\sim 3 \mathrm{~ns}$, which can be attributed to shot-to-shot experimental variations in the targets and laser energy. Simulated mass density reproduces the increase in density seen at the shock front in XRBL [see Figs. 2(a) and 2(d)], which can be attributed to regions of postshock xenon followed by $\mathrm{CH}-\mathrm{Br}$. The plots of materials indicate that the typical width of the postshock xenon is $\sim 40 \mu \mathrm{m}$, i.e., in the limit of the diagnostic resolution due to motion blurring. The simulations show the shock front as an unstable, rippled layer due to the growth of hydrodynamic instabilities mediated by strong radiative cooling in the shock which led to an increase in its density, thus making the interface with the upstream, unshocked xenon unstable. The simulated electron density in Fig. 2(d) at 22 ns shows significant heating ahead of the shock due to the formation of the radiative precursor.

As the XRBL diagnostic is sensitive to variations in mass density, it does not provide information on the radiative precursor which is characterized by changes in temperature and ionization. Thus, the radiative precursor was studied by measuring the electron density ahead of the shock with laser interferometry. Figure 3 shows results from 1D axial streak imaging [Figs. 3(a) and 3(b)] and 2D time-resolved GOI imaging [Figs. 3(c) and 3(d)]. The displacement of the interference fringes from their initial undisturbed position [the straight lines in Figs. 3(a) and 3(c)] is proportional to the line electron density $n_{e} L$, i.e., the electron density integrated along the length of the plasma being probed. As time progresses, the fringe contrast decreases and the displacement cannot be traced accurately as the laser goes through regions near the shocks, characterized by strong absorption and large spatial gradients of electron density. The resulting $n_{e} L$ maps [Figs. 3(b) and 3(d)] were obtained using the technique described in Ref. [34]. The streak interferometry results in Fig. 3(b) show that isocontours between $n_{e} L=1 \times 10^{18} \mathrm{~cm}^{-2}$ and $3.5 \times 10^{18} \mathrm{~cm}^{-2}$ maintain an approximately constant separation from $\sim 12-20 \mathrm{ns,}$ indicating that, during these times, the radiative precursor reaches a steady state with a characteristic extent of $\sim 300-350 \mu \mathrm{m}$. Such quasistationary radiative shocks have only been previously observed in 1D experiments [35] and in 2D numerical simulations [12]. By following a fixed value of $n_{e} L=1.5 \times 10^{18} \mathrm{~cm}^{-2}$, a characteristic precursor "velocity" of $\sim 90 \mathrm{~km} / \mathrm{s}$ is estimated, in agreement with the shock velocity estimated from the XRBL results.

Results from 2D $n_{e} L$ at 18 ns in Figs. 3(c) and 3(d) show features similar to those seen in XRBL results in Fig. 2(a); however, with this diagnostic, the shocks cannot be
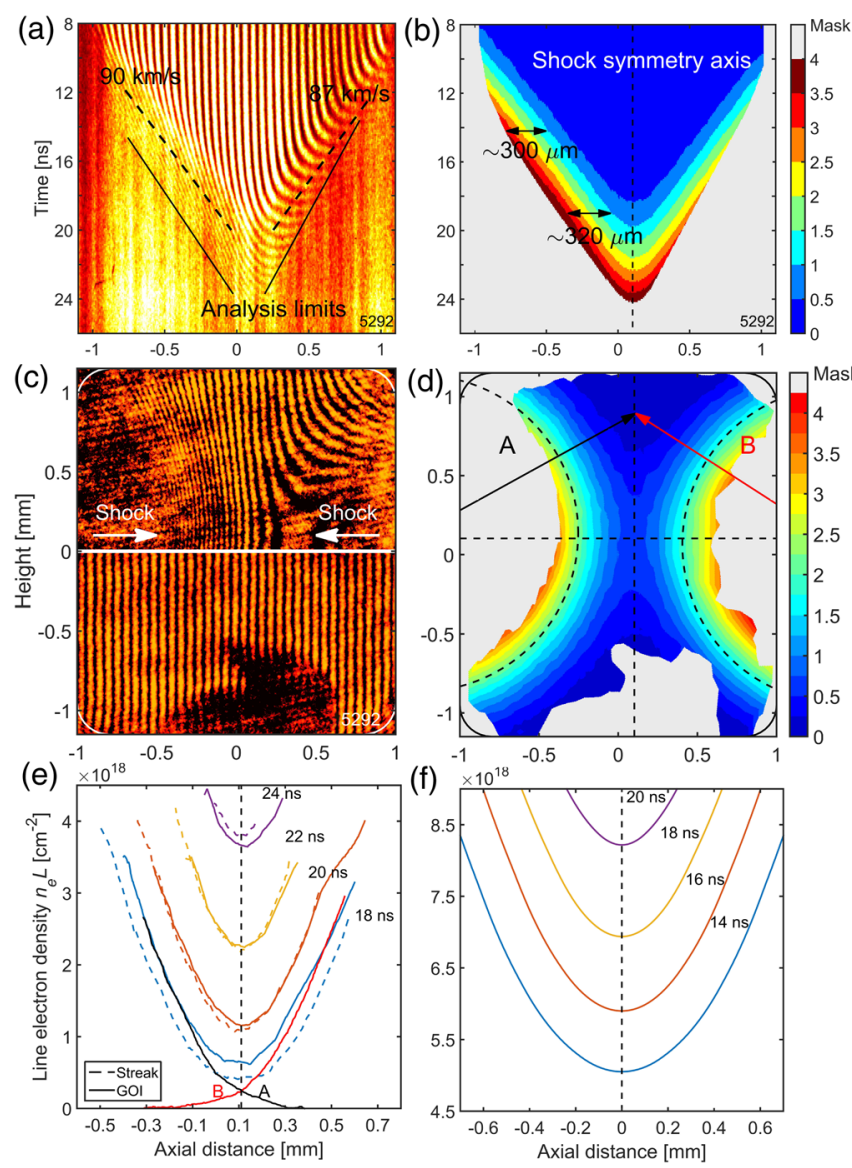

FIG. 3. Electron density measurements in the radiative precursors. (a),(b) 1D axial streak interferometry results with respect to (a) the raw data and (b) the analysis of (a), resulting in the line electron density $n_{e} L\left(\times 10^{18} \mathrm{~cm}^{-2}\right)$ as a function of time. The dashed lines in (a) mark values of $n_{e} L \sim 1.5 \times 10^{18} \mathrm{~cm}^{-2}$. (c),(d) $2 \mathrm{D}$ GOI results at $18 \mathrm{~ns}$. The top half of (c) shows raw data, and the bottom half a preshot interferogram. (d) 2D $n_{e} L$ map from an analysis of (c). (e) Axial profiles of $n_{e} L$ at 18, 20, 22, and $24 \mathrm{~ns}$ from (b) and (d). Also shown are $n_{e} L$ profiles at $18 \mathrm{~ns}$ off axis [the positions marked $A$ and $B$ in (d)]. (f) Simulated axial profiles of $n_{e} L$ at 14, 16, 18, and $20 \mathrm{~ns}$.

accurately resolved and are seen as diffuse regions. The analysis in Fig. 3(d) shows that the isocontours of $n_{e} L$ between 1 and $3 \times 10^{18} \mathrm{~cm}^{-2}$ can be well approximated as concentric circles (e.g., $n_{e} L=2 \times 10^{18} \mathrm{~cm}^{-2}$, shown as dashed circles), indicating that the precursors have a spherical shape at this particular time. This allows defining origins for radial axes of symmetry for both shocks, roughly aligned with the horizontal shock symmetry axis (shown as straight dashed lines). The radial symmetry allows extracting profiles of $n_{e} L$ towards regions off axis, where little interaction with the counterpropagating precursor is expected. Under this approximation, it is possible to extract the expected $n_{e} L$ profiles for a single-drive shock [labeled $A$ and $B$ in Fig. 3(d)].

Figure 3(e) shows a comparison of axial profiles of $n_{e} L$ from streak and GOI imaging at 18, 20, 22, and $24 \mathrm{~ns}$, 


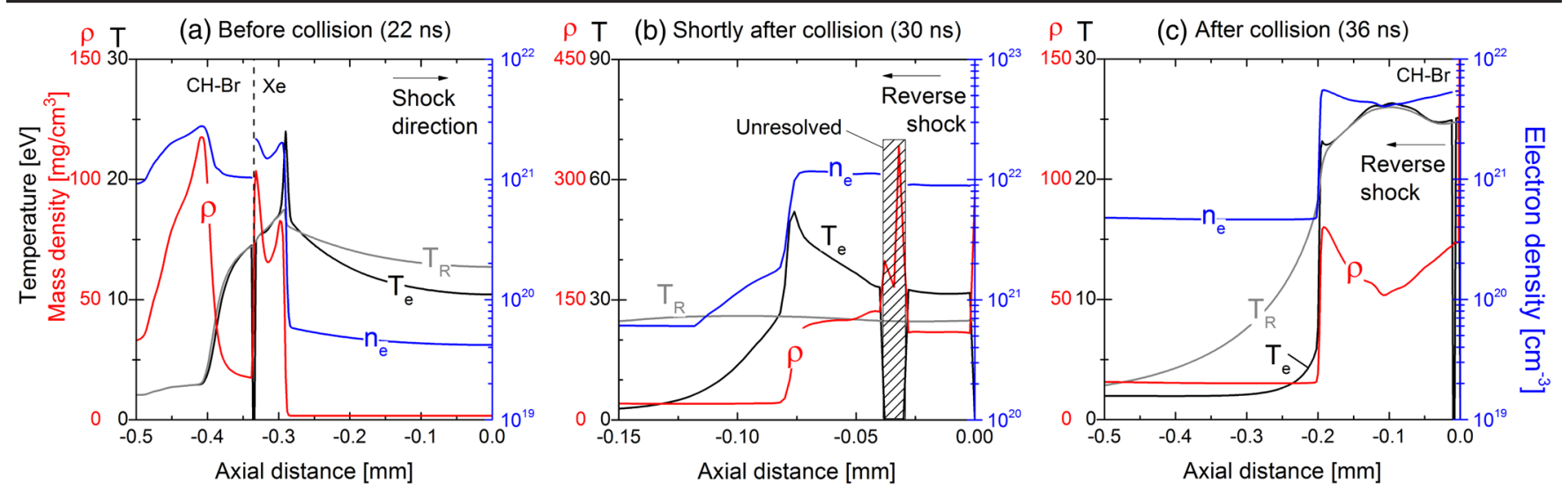

FIG. 4. Axial profiles of mass density $(\rho)$, electron density $\left(n_{e}\right)$, electron temperature $\left(T_{e}\right)$, and radiation temperature $\left(T_{R}\right)$ from $2 \mathrm{D}$ simulations of counterpropagating radiative shocks at: (a) $22 \mathrm{~ns}$ [before the collision; see Fig. 3(d)], (b) 30 ns (shortly after the collision, note the change in the $X$ and $Y$ scales), (c) 36 ns [after the collision; see Fig. 3(f)]. The axial distance is taken from the center of the diagnostic window, which marks the position of a reflective boundary used for the simulations.

showing very good agreement between both diagnostics. Discrepancies are probably due to uncertainties in defining a "zero" level of $n_{e} L$ [34] in the analysis of GOI data. Comparison between the profiles on and off axis at $18 \mathrm{~ns}$ in Fig. 3(e) show the radiative precursors have very similar values away from the vertical symmetry axis and, as they reach the collision in the center, it leads to an effective increase in $n_{e} L$. This increase is consistent with the values obtained by doing the sum between the two off-axis profiles $A$ and $B$.

The axial spatial distribution of $n_{e} L$ from the two radiative precursors in Fig. 3(e) can be compared to results from 2D simulations shown in Fig. 3(f). The simulations overall match the spatial distribution of $n_{e} L$ in the experiments with a time difference of $4 \mathrm{~ns}$ (14-20 ns in simulations, 18-24 ns in the experiments). Moreover, simulations overestimate the experimental values of $n_{e} L$ by a constant value of $\sim 4.5 \times 10^{18} \mathrm{~cm}^{-3}$, which is consistent with experiments having a 3D distribution of electron density, rather than the $2 \mathrm{D}$ one used in the simulations; thus, lower values should be expected in reality, as discussed in Ref. [36].

In order to get a better understanding of the collision between the two counterpropagating radiative shocks, axial profiles from 2D numerical simulations in Figs. 4(a)-4(c) show, respectively, the plasma conditions at 22 ns (precollision), at $30 \mathrm{~ns}$ (shortly after the collision), and at $36 \mathrm{~ns}$ (postcollision). In Fig. 4(a), the dip in electron temperature at $\sim-0.34 \mathrm{~mm}$ from the reflective boundary (at $0 \mathrm{~mm}$ ) marks regions of $\mathrm{CH}-\mathrm{Br}$ and $\mathrm{Xe}$, whereas the peak in electron temperature of $T_{e} \sim 24 \mathrm{eV}$ at $\sim-0.29 \mathrm{~mm}$ marks the position of the shock front. The radiative precursor is seen ahead of the shock with a peak temperature of $T_{e} \sim 16 \mathrm{eV}$, decreasing to $T_{e} \sim 10 \mathrm{eV}$ on the axis of the window. Similar electron temperatures pre- and postshock indicate the shock is supercritical [3]. The postshock xenon temperature $T_{\mathrm{ps}}$ agrees with estimates presented in Ref. [11] done by balancing the fluxes of radiation and kinetic energy of the incoming flow $\left(2 \sigma T_{\mathrm{ps}}^{4}=\rho_{0} v_{\mathrm{s}}^{3} / 2\right)$. Here, $\rho_{0}=$ $1.6 \mathrm{mg} / \mathrm{cm}^{3}$ is the initial Xe density, $v_{\mathrm{s}} \sim 80 \mathrm{~km} / \mathrm{s}$ is the measured shock velocity and $\sigma=5.67 \times 10^{-8} \mathrm{~W} \mathrm{~m}^{-2} \mathrm{~K}^{-4}$ is the Stefan-Boltzmann constant, resulting in $T_{\mathrm{ps}} \sim 20 \mathrm{eV}$.

The simulated mass density in the postshock xenon region in Fig. 4(a) at 22 ns shows a double peak which reflects the spatial variations in density due to the formation of hydrodynamic instabilities seen in 2D images (ripples). Thus, a lower boundary for the postshock compression can be estimated by taking the density at the through (which remains constant between 22-26 ns) of $\rho_{\mathrm{ps}} \sim 60 \mathrm{mg} / \mathrm{cm}^{3}$, resulting in a compression of $\rho_{\mathrm{ps}} / \rho_{0} \sim 38$. On a first approximation, the postshock compression can also be estimated experimentally from the XRBL results in Fig. 2(a) by taking the ratio of absorbed X-ray intensity at a point through the postshock $I_{\mathrm{ps}}$ with respect to the intensity through the undisturbed ambient xenon $I_{0}$ via the expression $\rho_{\mathrm{ps}} / \rho_{0}=1+\left[\ln \left(I_{0} / I_{\mathrm{ps}}\right) /\left(\sigma_{\mathrm{Xe}} L_{\mathrm{Xe}} \rho_{0}\right)\right]$, where $\sigma_{\mathrm{Xe}}=505 \mathrm{~cm}^{2} / \mathrm{g}$ is the mass attenuation coefficient for $\mathrm{Xe}$ at $6.7 \mathrm{keV}$ and $L_{\mathrm{Xe}} \sim 0.3 \mathrm{~mm}$ is the transverse length of the xenon postshock. Typical values of $I_{0} / I_{\mathrm{ps}}$ are $\sim 1.1$, and thus from different shots $\rho_{\mathrm{ps}} / \rho_{0} \sim 6 \pm 2$. It should be noted that this estimate is heavily constrained by the resolution of the XRBL diagnostic (on the order of the extent of the postshock region) and the possible emission of hard $\mathrm{x}$ rays $(>10 \mathrm{keV})$ from the backlighter [37] which could affect the intensity measurements. Overall, the compressions that characterize the postshock are higher than the ideal, nonradiative compression of $4 \times$ [19], which indicates that radiative losses play a significant role in the shock dynamics.

Results from simulations postcollision, e.g., from $28 \mathrm{~ns}$ onwards [Figs. 4(b) and 4(c) and Figs. 2(e) and 2(f)], indicate that the reverse shock is formed mostly of piston material (CH-Br) with an almost unresolved xenon region close to the reflective boundary. The postshock density in the reverse 
shock $\rho_{\text {prs }}$ can be estimated by using the Rankine-Hugoniot relations for a reverse shock $[28,38]$ as $\rho_{\text {prs }}=\left[\left(v_{\mathrm{rs}}+\right.\right.$ $\left.\left.v_{\mathrm{ps}}\right) / v_{\mathrm{rs}}\right] \rho_{\mathrm{ps}}$, where $v_{\mathrm{rs}}$ is the reverse shock velocity in the laboratory frame (measured as $v_{\mathrm{rs}} \sim 30 \mathrm{~km} / \mathrm{s}$ ), $v_{\mathrm{ps}}$ is the postshock velocity in the laboratory frame given by $v_{\mathrm{ps}}=$ $v_{\mathrm{s}}\left(\rho_{\mathrm{ps}}-\rho_{0}\right) / \rho_{\mathrm{ps}} \approx 73 \mathrm{~km} / \mathrm{s}$ for a postshock mass density from simulations of $\rho_{\mathrm{ps}} \sim 60 \mathrm{mg} / \mathrm{cm}^{3}$, resulting in $\rho_{\text {prs }} \sim 206 \mathrm{mg} / \mathrm{cm}^{3}$. This estimate is in line with simulation results at 28-30 ns [see, e.g., Fig. 4(b)], which indicate that $\rho_{\text {prs }} \sim 110-190 \mathrm{mg} / \mathrm{cm}^{3}$, with the caveat that strong mixing between $\mathrm{Xe}$ and $\mathrm{CH}-\mathrm{Br}$ should be predominant at these times. Simulations at $36 \mathrm{~ns}$ in Fig. 4(c) show the reverse shock as an extended region with a half-width of $\sim 0.2 \mathrm{~mm}$ and with an approximately constant mass density and temperature of $\rho \sim 50 \mathrm{mg} / \mathrm{cm}^{3}$ and $T_{e} \sim 25 \mathrm{eV}$, respectively. This region drives a strong reverse-radiative precursor evidenced by an increased radiation temperature $T_{R}$.

In summary, we presented a new study of laser-piston driven radiative shocks in xenon characterized by simultaneous experimental measurements of the dynamics of the shock region and the radiative precursor. This experimental setup allowed us to study the collision between two counterpropagating radiative shocks as a radiation-hydrodynamics platform to study complex physics, and the setup is particularly well suited for numerical benchmarking. Simulations are able to accurately reproduce the experimental results, and we hope the first results in this Letter can be used as a test bed for other codes (e.g., 3D radiative codes) to investigate, for instance, the effect of the interaction between the precursors and the formation of the reverse shocks. Similarly, we intend for future experiments to corroborate the estimates presented here by measuring the precursor and postshock plasma conditions in xenon with, e.g., x-ray Thomson scattering, which to date has been done only for radiative shocks in argon [39].

This work was supported by STFC and AWE through their academic access program and, in part, by The Royal Society, EPSRC and Labex PLAS@PAR. The authors would like to acknowledge Robert Charles, Jim Firth, Paul Treadwell, Rob Johnson, David Hillier, Nick Hopps, and the entire Orion team for their help and support during the experiments.

*f.suzuki@imperial.ac.uk

†Present address: Lawrence Livermore National Laboratory, Livermore, CA 94550, USA.

${ }^{\ddagger}$ Present Address: Extreme Light Infrastructure (ELI), Institute of Plasma Physics, Czech Academy of Sciences, 182 00 Prague 8, Czech Republic.

${ }^{\S}$ Present address: First Light Fusion Ltd., Unit 10, Oxford Industrial Park, Mead Road, Yarnton, Oxfordshire OX5 1QU, United Kingdom.
"Also at Blackett Laboratory, Imperial College London, London SW7 2BW, United Kingdom.

Present address: Lawrence Livermore National Laboratory, Livermore, CA 94550, USA.

[1] J. M. Blondin, E. B. Wright, K. J. Borkowski, and S. P. Reynolds, Astrophys. J. 500, 342 (1998).

[2] P. Hartigan, Astrophys. Space Sci. 287, 111 (2003).

[3] Y. B. Zel'dovich and Y. P. Raizer, Physics of Shock Waves and High-Temperature Hydrodynamic Phenomena, 1st ed. (Academic Press, New York, 1966).

[4] R. P. Drake, L. Davison, and Y. Horie, High Energy Density Physics (Springer, New York, 2006).

[5] R. G. McClarren, R. P. Drake, J. E. Morel, and J. P. Holloway, Phys. Plasmas 17, 093301 (2010).

[6] D. E. Innes, J. R. Giddings, and S. A. E. G. Falle, Mon. Not. R. Astron. Soc. 224, 179 (1987).

[7] S. Leygnac, L. Boireau, C. Michaut, T. Lanz, C. Steh, C. Clique, and S. Bouquet, Phys. Plasmas 13, 113301 (2006).

[8] M. González, E. Audit, and C. Stehlé, Astron. Astrophys. 497, 27 (2009).

[9] B. van der Holst, G. Tóth, I. V. Sokolov, L. K. S. Daldorff, K. G. Powell, and R. P. Drake, High Energy Density Phys. 8, 161 (2012).

[10] B. Fryxell, E. Rutter, and E. S. Myra, High Energy Density Phys. 8, 141 (2012).

[11] R. P. Drake et al., High Energy Density Phys. 7, 130 (2011).

[12] C. Michaut, E. Falize, C. Cavet, S. Bouquet, M. Koenig, T. Vinci, A. Reighard, and R. P. Drake, Astrophys. Space Sci. 322, 77 (2009).

[13] J. F. Hansen, M. J. Edwards, D. H. Froula, G. Gregori, A. D. Edens, and T. Ditmire, Phys. Plasmas 13, 022105 (2006).

[14] A. D. Edens, R. G. Adams, P. Rambo, L. Ruggles, I. C. Smith, J. L. Porter, and T. Ditmire, Phys. Plasmas 17, 112104 (2010).

[15] M. J. Edwards, A. J. MacKinnon, J. Zweiback, K. Shigemori, D. Ryutov, A. M. Rubenchik, K. A. Keilty, E. Liang, B. A. Remington, and T. Ditmire, Phys. Rev. Lett. 87, 085004 (2001).

[16] A. S. Moore, J. Lazarus, M. Hohenberger, J. S. Robinson, E. T. Gumbrell, M. Dunne, and R. A. Smith, Astrophys. Space Sci. 307, 139 (2007).

[17] M. Hohenberger, D. R. Symes, J. Lazarus, H. W. Doyle, R. E. Carley, A. S. Moore, E. T. Gumbrell, M. M. Notley, R. J. Clarke, M. Dunne, and R. A. Smith, Phys. Rev. Lett. 105, 205003 (2010).

[18] J. C. Bozier, G. Thiell, J. P. Le Breton, S. Azra, M. Decroisette, and D. Schirmann, Phys. Rev. Lett. 57, 1304 (1986).

[19] S. Bouquet, C. Stéhlé, M. Koenig, J.-P. Chièze, A. BenuzziMounaix, D. Batani, S. Leygnac, X. Fleury, H. Merdji, C. Michaut, F. Thais, N. Grandjouan, T. Hall, E. Henry, V. Malka, and J.-P. J. Lafon, Phys. Rev. Lett. 92, 225001 (2004).

[20] X. Fleury, S. Bouquet, C. Stehle, M. Koenig, D. Batani, A. Benuzzi-Mounaix, J.-P. Chieze, N. Grandjouan, J. Grenier, T. Hall, E. Henry, J.-P. Lafon, S. Leygnac, V. Malka, B. Marchet, H. Merdji, C. Michaut, and F. Thais, Laser Part. Beams 20, 263 (2002).

[21] A. B. Reighard, R. P. Drake, K. K. Dannenberg, D. J. Kremer, M. Grosskopf, E. C. Harding, D. R. Leibrandt, 
S. G. Glendinning, T. S. Perry, B. A. Remington, J. Greenough, J. Knauer, T. Boehly, S. Bouquet, L. Boireau, M. Koenig, and T. Vinci, Phys. Plasmas 13, 082901 (2006).

[22] M. González, C. Stehlé, E. Audit, M. Busquet, B. Rus, F. Thais, O. Acef, P. Barroso, A. Bar-Shalom, D. Bauduin, M. Kozlovà, T. Lery, A. Madouri, T. Mocek, and J. Polan, Laser Part. Beams 24, 535 (2006).

[23] C. Stehlé, M. Kozlová, J. Larour, J. Nejdl, N. Champion, P. Barroso, F. Suzuki-Vidal, O. Acef, P.-A. Delattre, J. Dostál, M. Krus, and J.-P. Chièze, Opt. Commun. 285, 64 (2012).

[24] F. W. Doss, H. F. Robey, R. P. Drake, and C. C. Kuranz, Phys. Plasmas 16, 112705 (2009).

[25] C. M. Krauland, R. P. Drake, C. C. Kuranz, B. Loupias, T. Plewa, C. M. Huntington, D. N. Kaczala, S. Klein, R. Sweeney, R. P. Young, E. Falize, B. Villette, and P. A. Keiter, Astrophys. J. 762, L2 (2013).

[26] M. Koenig, T. Vinci, A. Benuzzi-Mounaix, N. Ozaki, a. Ravasio, M. Rabec le Glohaec, L. Boireau, C. Michaut, S. Bouquet, S. Atzeni, A. Schiavi, O. Peyrusse, and D. Batani, Phys. Plasmas 13, 056504 (2006).

[27] P. Velarde, D. García-Senz, E. Bravo, F. Ogando, A. Relańo, C. García, and E. Oliva, Phys. Plasmas 13, 092901 (2006).

[28] A. Pak et al., Phys. Plasmas 20, 056315 (2013).

[29] N. Hopps et al., Plasma Phys. Controlled Fusion 57, 064002 (2015).
[30] C. C. Kuranz, B. E. Blue, R. P. Drake, H. F. Robey, J. F. Hansen, J. P. Knauer, M. J. Grosskopf, C. Krauland, and D. C. Marion, Rev. Sci. Instrum. 77, 10E327 (2006).

[31] D. W. Phillion and C. J. Hailey, Phys. Rev. A 34, 4886 (1986).

[32] P. D. Roberts, S. J. Rose, P. C. Thompson, and R. J. Wright, J. Phys. D 13, 1957 (1980).

[33] D. L. Youngs, Physica (Amsterdam) 12D, 32 (1984).

[34] G. F. Swadling, S. V. Lebedev, N. Niasse, J. P. Chittenden, G. N. Hall, F. Suzuki-Vidal, G. Burdiak, A. J. Harvey-Thompson, S. N. Bland, P. De Grouchy, E. Khoory, L. Pickworth, J. Skidmore, and L. Suttle, Phys. Plasmas 20, 022705 (2013).

[35] C. Stehlé, M. González, M. Kozlova, B. Rus, T. Mocek, O. Acef, J. P. Colombier, T. Lanz, N. Champion, K. Jakubczak, J. Polan, P. Barroso, D. Bauduin, E. Audit, J. Dostal, and M. Stupka, Laser Part. Beams 28, 253 (2010).

[36] T. Vinci, M. Koenig, A. Benuzzi-Mounaix, N. Ozaki, A. Ravasio, L. Boireau, C. Michaut, S. Bouquet, S. Atzeni, A. Schiavi, and O. Peyrusse, J. Phys. IV (France) 133, 1039 (2006).

[37] C. M. Krauland, L. C. Jarrott, R. P. Drake, P. A. Keiter, C. C. Kuranz, B. Westover, H. Sawada, D. N. Kaczala, and P. Bonofiglo, Rev. Sci. Instrum. 83, 10E528 (2012).

[38] C. Fortmann, H. J. Lee, T. Döppner, R. W. Falcone, A. L. Kritcher, O. L. Landen, and S. H. Glenzer, Phys. Rev. Lett. 108, 175006 (2012).

[39] A. J. Visco, R. P. Drake, S. H. Glenzer, T. Döppner, G. Gregori, D. H. Froula, and M. J. Grosskopf, Phys. Rev. Lett. 108, 145001 (2012). 